First symptom of onset was Raynaud phenomena $91.3 \%$ of the cases, followed by skin changes $(56.5 \%)$, puffy fingers $(52,2 \%)$, gastrointestinal and musculoarticular symptoms $(23.9 \%$ each).

The medium duration between the first symptom and a medical consult was 6 (63.5) months. The first medical consult was done by a internal medicine specialist $-38.3 \%$, by a rheumatologist- $29.8 \%$, a gastroenterologist- $12.8 \%$, a dermatologist- $8.5 \%$, a nephrologist and a pneumologist $-4.2 \%$ and neurologist $2.1 \%$

The first suspected diagnosis was SSc in 14 cases, Raynaud syndrome in 8 , connective tissue disease in 5 , rheumatoid arthritis in 4 , cancer, autoimmune hepatitis, idiopathic pulmonary fibrosis each in 2 cases and none in 10 cases. The medium number of consults until final diagnosis was 3.4 (1.7). The medium duration from the first symptom until the correct diagnosis was 39.2 (74) months. The first investigations recommended were blood tests in almost all of the patients $(95.7 \%)$, but only a third of them included specific scleroderma autoantibodies. Capillaroscopy was performed as an initial diagnostic test in only 6 patients $(12.8 \%)$. The mean interval from disease onset until the patient was referred to the first capillaroscopy was 13.5 (28.8) months, to specific autoantibodies was 40.17 (61.3) month, to echocardiography was 36.38 (54) months, to lung function tests and lung CT - 41.76 (65.8) months.

There were no significant statistical differences between patients coming from rural environment and those coming from urban environment. The only significant statistical difference between diffuse and limited subset was the time the patient was referred to echocardiography (19.8 (47.6) months for the diffuse subset, 66.8 (94.6) months for the limited subset, $p=0.04)$.

The only statistical difference between males and females was related to the interval that capillaroscopy was performed (14 (20.5)months in females, 4.8 (5) months in males, $\mathrm{p}=0.02$ ).

Conclusions: Scleroderma is a less well-known disease. This lack of awareness contributes to delayed diagnosis and delayed onset of therapy. Often such diagnostic uncertainty and frustration takes a huge toll on the psychological well-being of these patients, who describe their journey to diagnosis as being one of the most difficult part of their illness. One of our missions as rheumatologist is to increase recognition of this disorder.

References:

[1] https://www.sclero.org/scleroderma/diagnosis/difficult.

Disclosure of Interest: None declared

DOI: 10.1136/annrheumdis-2017-eular.3954

\section{AB0648 DISEASE-RELATED AUTOANTIBODY PROFILE IN SYSTEMIC SCLEROSIS IN GREECE}

C. Liaskos ${ }^{1}$, E. Marou ${ }^{1}$, T. Simopoulou ${ }^{1}$, M. Barmakoudi ${ }^{1}$, G. Efthymiou ${ }^{1}$, T. Scheper ${ }^{2}$, W. Meyer ${ }^{2}$, D.P. Bogdanos ${ }^{1}$, L. Sakkas ${ }^{1} .{ }^{1}$ Rheumatology and Clinical Immunology, University of Thessaly, School of Health Sciences, LARISSA, Greece; ${ }^{2}$ Institute of Immunology, Euroimmun AG, Lubeck, Germany

Background: Autoantibodies (autoAbs) help in diagnosis and predicting clinical phenotypes in systemic sclerosis (SSc).

Objectives: To determine the clinical utility of 13 SSc-related autoAbs in SSc patients in Greece.

Methods: 131 consecutive patients with SSc (111 female, mean age $58.1 \pm 14$ years; 49 with diffused cutaneous SSc [dcSSc] and 82 with limited cutaneous SSc [IcSSc]) were analyzed by a multiplex line immunoassay (Euroimmun) for autoAbs against 13 SSc-related antigens. Twenty two patients with primary Raynaud phenomenon (RP), and 22 healthy controls were also analyzed

Results: ANA by indirect immunofluorescence was present in $128(97.7 \%)$ patients with SSc. Excluding anti-Ro52, 113 (89.3\%) SSc patients were positive for at least one autoAb: anti-Topo I abs in $54(41.2 \%)$, anti-CENPA in $37(28.2 \%$, all reactive with CENPB), anti-RNA polymerase III (RP11) in 19 (14.5\%), anti-RNA polymerase III (RP155) in $13(9.9 \%)$, anti-fibrillarin in $4(3.1 \%)$, anti-Ku in $6(4.6 \%)$, anti-NOR90 in $8(6.1 \%)$, anti-PM/Scl100 in $2(1.5 \%)$, and anti-PM/Scl75 in 4 (3.1\%). There was no immunoreactivity for Th/To or PDGFR. Overall, 102 (77.9\%) SSc patients had autoAbs against Topo I, CENPA or CENPB, RP11 or RP155. Anti-Topo I abs were strongly associated with dcSSc, interstitial lung disease (ILD) $(p<0.001)$, and pulmonary arterial hypertension (PAH) $(p=0.019)$. Anti-CENB abs were associated with IcSSc, and negatively associated with ILD. Anti-RP11 and anti-NOR90 abs were associated with male gender, and anti-NOR90 associated with ILD. In PR individuals and healthy individuals anti-Ro52 immunoreactivity was found in $9.1 \%$ and $13.6 \%$, respectively, but no other immunoreactivity was detected.

Conclusions: Anti-Scl70, anti-CENP and anti-RNA pol III are the most prevalent autoAbs in SSc. Anti-Topo I and anti-NOR90 abs are associated with ILD and/or PAH.

Disclosure of Interest: C. Liaskos: None declared, E. Marou: None declared, T. Simopoulou: None declared, M. Barmakoudi: None declared, G. Efthymiou: None declared, T. Scheper Employee of: Euroimmun AG, W. Meyer Employee of: Euroimmun AG, D. Bogdanos: None declared, L. Sakkas: None declared DOI: 10.1136/annrheumdis-2017-eular.4641

\section{AB0649 SYSTEMIC SCLEROSIS IN ARGENTINA: EVALUATION OF A SINGLE CENTER COHORT AND COMPARISON WITH INTERNATIONAL SERIES}

M. Garcia Carrasco ${ }^{1}$, L.O. Roa Prerez ${ }^{1,2}$, R. Gomez ${ }^{1,2}$, S. Leal ${ }^{1,2}$, F. Paniego ${ }^{1}$, N. Perez ${ }^{1}$, G.G. Nasswetter ${ }^{1}$, D. Diana ${ }^{1} .{ }^{1}$ Rheumatology Division; ${ }^{2}$ Hospital de Clinicas José de San Martín, Buenos Aires, Argentina

Background: Systemic sclerosis (SS) is an autoimmune disease with generalized vascular dysfunction and a myriad of clinical and laboratory manifestations. Spreading of thickening of the skin, serological characteristics and the pattern of compromise of the internal organs help to classify them as diffuse scleroderma, localized sclerosis and systemic sinus scleroderma. Reports of Latin American cohorts published to date are scarce.

Objectives: To describe the clinical and serological data for a single center cohort and to compare those with national and international cohorts.

Methods: Descriptive, observational, cross-sectional study. We analyzed our SS database. Patients were evaluated since $01 / 15$ to $05 / 2016$ and fulfilled SS classification criteria (ACR 1980/ACR-EULAR 2013).Patients were classified according to Le Roy criteria in Limited Systemic Sclerosis (ISS), Diffuse Systemic Sclerosis (dSS), Systemic Sclerosis sine scleroderma (SsnS) and pre-systemic sclerosis (pSS). Organic compromise was defined as follows: Gastrointestinal (GI): esophagic dismotility by manometry or esophagitis by endoscopy; Lung: Pulmonary hypertension $(\mathrm{PH})$ : PSAP $>25 \mathrm{mmHg}$ by right heart catheterization; Intersticial Lung Disease (ILD): compatible HRCT or $<70 \%$ predicted FVC or $<80 \%$ predicted DLCO; Heart: Left ventricle dysfunction without PH or arterial hypertension, pericardial effusion; Digital ulcers and pitting scars; History of sclerodermic renal crisis (RSC); calcinosis. Anti-Scl-70 was determined by ELISA and anti-Centromere (ACA) by IIF.

Results: 123 patients were included. $74 \%$ with ISS, $24 \%$ dSS and $2 \%$ SsnS; age at diagnosis (years): 49.7 (18-79 DS: 12.27) and 48.7 (27-79 DS: 13.01) in ISS and dSS respectively. Raynaud's phenomenon previous to diagnosis (years) 7,7 (0-54 DS: 12.81 ) in ISS and 4.03 (0-22 DS: 5.5 ) in dSS. $49.5 \%$ ISS were ACA positive vs $17 \%$ in dSS as well also $41,4 \%$ had Scl-70 positive $(p<0.05)$. Lung: $58.6 \%$ of dSS presented ILD and $3,4 \%$ RSC, according to published reports. No statistically significant differences were found about the presence of calcinosis, digital tip ulcers or PH between dSS and ISS. GI: was studied in only 39/123 patients with $84,6 \%$ affected among those.

Table 1. Comparison of main variables among different cohorts

\begin{tabular}{|c|c|c|c|c|c|c|c|c|c|c|}
\hline & \multicolumn{4}{|c|}{ Diffuse } & \multicolumn{6}{|c|}{ Limited systemic sclerosis } \\
\hline & \multicolumn{2}{|c|}{ US, $n$} & \multicolumn{2}{|c|}{ EUSTAR, $n$} & \multicolumn{2}{|c|}{ Spain, $\mathrm{n}$} & \multicolumn{2}{|c|}{ Germany, n } & \multicolumn{2}{|c|}{ Argentina ${ }^{\star}, n$} \\
\hline & 119 & 128 & 2838 & 4481 & 243 & 568 & 484 & 674 & 29 & 91 \\
\hline $\begin{array}{l}\text { Oesophageal } \\
\text { dysmotility (\%) }\end{array}$ & 67 & 67 & 70 & 66 & 71 & 57 & 69 & 59 & 31.5 & 38.5 \\
\hline ILD (\%) & 63 & 37 & 52 & 11 & 70 & 39 & 56 & 21 & 59 & 22 \\
\hline $\mathrm{PH}(\%)$ & 2 & 31 & 6 & ND & 14 & 9 & 19 & 15 & 3.4 & 3.3 \\
\hline RSC (\%) & 17 & 2 & 4 & 1 & 8 & 1 & 16 & 9 & 3 & 0 \\
\hline Digital ulcers (\%) & ND & ND & 20 & 33 & 64 & 39 & 34 & 24 & 10.3 & 5.5 \\
\hline Calcinosis (\%) & 23 & 42 & ND & ND & 24 & 20 & ND & ND & 14 & 19 \\
\hline
\end{tabular}

Calcinosis $(\%)$

${ }^{*} \mathrm{HCJSM}$.

Conclusions: Our data showed similar results to the local and european cohorts. We found fewer calcinosis and digital tip ulcers in both ISS and dSS than international cohorts. A lower prevalence of $\mathrm{PH}$ was reported in ISS respect to other series, and it might be atributed to the method wich it was measured. (right heart catheterism).

Disclosure of Interest: None declared

DOI: 10.1136/annrheumdis-2017-eular.3520

\section{AB0650 ANTI-TOPOISOMERASE POSITIVE SYSTEMIC SCLEROSIS PROGNOSIS INFAUST?}

M. Boonstra, G.A. Beerends, H.U. Scherer, J.K. de Vries-Bouwstra. Rheumatology, LUMC, Leiden, Netherland's

Background: Anti-topoisomerase antibodies (ATA) in systemic sclerosis (SSc) have been associated with poorer prognosis including diffuse skin involvement, pulmonary fibrosis, cardiac involvement and increased mortality. However, 30$60 \%$ of ATA-positive SSc patients demonstrate only limited skin involvement and some have only mild disease course. In SSc, optimal risk stratification is of utmost importance for tailored clinical management at the patient level.

Objectives: We aimed to determine the prevalence of mild disease among ATA positive patients and to investigate which readily available clinical parameters best identify patients with highest disease severity.

Methods: Clinical baseline data from SSc patients included in the Combined Care In Systemic Sclerosis (CCIS) cohort of the Leiden University Medical Center were extracted. Patients fulfilling ACR 2013 criteria and ATA positive were included. Descriptive statistics were used to summarize sociodemographic, clinical and serological features. Patients with grade 3 or 4 disease on any of the Medsger severity subscales were considered to be severely diseased. We compared presence of diffuse cutaneous involvement, Raynaud's, pitting scars, calcinosis, proximal muscle weakness, $>10 \%$ weight loss, interstitial lung disease and pro-BNP between the severity groups and corrected for confounding by disease duration (time since non-Raynaud) by stratifying into quartiles. 